\title{
Mesurer les changements autodéclarés relatifs à la consommation d'alcool et de cannabis au cours de la deuxième vague de la pandémie de COVID-19 au Canada
}

\author{
Mélanie Varin, M. Sc.; Kate Hill MacEachern, Ph. D; Nousin Hussain, M.P.H.; Melissa M. Baker, Ph. D.
}

(Publié en ligne le 27 septembre 2021)

Diffuser cet article sur Twitter

\section{Résumé}

Cette étude présente des estimations représentatives à l'échelle nationale portant sur les changements autodéclarés relatifs à la consommation d'alcool et de cannabis depuis le début de la pandémie de COVID-19 au Canada. Nous nous sommes servi de données de l'Enquête sur la COVID-19 et la santé mentale (qui a eu lieu de septembre à décembre 2020) pour calculer la prévalence de ces changements. Les résultats révèlent que 15,7 \% des répondants ont déclaré avoir augmenté leur consommation d'alcool et 5,4\% leur consommation de cannabis depuis le début de la pandémie. Des disparités sociodémographiques ont été observées, laissant supposer que l'augmentation de la consommation d'alcool et de cannabis serait plus répandue au sein de certaines populations.

\section{Introduction}

Le Canada a confirmé un premier cas du nouveau coronavirus (le virus responsable de la COVID-19) le 25 janvier 2020. Début mars 2020, il était clair qu'une transmission communautaire s'était installée. Depuis, des directives et mesures de santé publique rigoureuses, comme le lavage des mains, le port obligatoire du masque, la fermeture des écoles et des protocoles de distanciation physique ont été mis en place au Canada. Ces mesures de santé publique ont joué un rôle essentiel dans l'atténuation de la propagation de la COVID-19 afin de protéger la santé des Canadiens. Or un nombre croissant de données probantes indique que la pandémie et les mesures strictes de santé publique qui en ont découlé ont eu un effet négatif sur la santé mentale et le bien-être des Canadiens ${ }^{1-3}$.

Les données recueillies au cours de la première vague de la pandémie de COVID-19 au Canada font état d'un plus grand nombre de personnes souffrent de stress, d'anxiété et de dépression ${ }^{1-5}$, des facteurs de risques reconnus comme étant associés à une consommation excessive et soutenue de substances comme l'alcool ${ }^{6-8}$ et le cannabis ${ }^{9}$. Selon une étude réalisée par Recherche en santé mentale Canada (RSMC), le tiers des participants âgés de 18 ans et plus qui ont déclaré avoir déjà reçu un diagnostic d'anxiété (n = 307) ou de dépression $(n=325)$ ont également déclaré avoir augmenté leur consommation d'alcool et de cannabis pendant la pandémie 4 .

De plus, des données recueillies entre le 29 mars et le 3 avril 2020 dans le cadre d'une enquête nationale réalisée auprès de 4383 participants âgés de 25 ans et plus indiquent que $14 \%$ des répondants ont déclaré avoir augmenté leur consommation d'alcool et 5,5 \% leur consommation de cannabis au cours de la première vague de la pandémie ${ }^{10}$. Les résultats obtenus auprès des adultes canadiens au début de la deuxième vague (du 14 au 21 septembre 2020) sont comparables : $40 \%$ des 3027 participants d'une étude ont déclaré que leur santé mentale s'est détériorée depuis le début de la pandémie $^{11}$. Au cours de la même période, la consommation d'alcool et de cannabis a

\section{Points saillants}

- Entre le 11 septembre et le 4 décembre 2020, 15,7 \% des répondants ont déclaré avoir augmenté leur consommation d'alcool et 5,4\% leur consommation de cannabis par rapport à avant la pandémie.

- Les personnes ayant déclaré que leur santé mentale était pire au moment de l'Enquête qu'avant la pandémie offraient la prévalence la plus forte en matière d'augmentation de la consommation d'alcool et de cannabis.

- Il est primordial de comprendre les déterminants sociaux de la santé pour élaborer des stratégies de réduction des méfaits et d'atténuation.

d'ailleurs augmenté de respectivement $30 \%$ et $20 \%$ chez les personnes qui souffraient déjà d'un problème de santé mentale $^{11}$. Ces constatations mettent en exergue la relation complexe entre santé mentale et consommation de substances.

Ces résultats révèlent un problème important et urgent à régler en matière de santé publique, la pandémie semblant avoir eu des répercussions à grande échelle sur les comportements en matière de santé ${ }^{12}$. Le Canada cherchant à élaborer un plan de relance nationale pour faire face aux répercussions et aux conséquences à long terme de la pandémie de COVID-19, davantage de données nationales sur les comportements en matière de santé, notamment sur la consommation de substances, 
sont nécessaires afin d'orienter les directives de santé publique, ces dernières prévoyant par exemple la création de messages de santé publique axés sur l'atténuation des méfaits associés à la consommation d'alcool et de cannabis. On dispose actuellement de peu d'information sur la consommation d'alcool et de cannabis à l'échelle nationale pendant la deuxième vague de la pandémie au Canada. L'objectif de cet article est (1) de quantifier approximativement les changements autodéclarés relatifs à la consommation d'alcool et de cannabis pendant la deuxième vague de la pandémie de COVID-19 et (2) de ventiler les données sur l'augmentation de la consommation d'alcool et de cannabis en fonction de caractéristiques sociodémographiques et des changements autodéclarés en matière de santé mentale.

\section{Méthodologie}

\section{Collecte de données et échantillonnage}

Les données utilisées pour cette étude proviennent de l'Enquête sur la COVID-19 et la santé mentale (ECSM), une enquête transversale élaborée et financée par Statistique Canada et l'Agence de la santé publique du Canada (ASPC). Cette enquête a été réalisée auprès de 30000 ménages entre le 11 septembre et le 4 décembre 2020 dans le but de recueillir de l'information sur la santé mentale et le bien-être de la population. Un échantillon aléatoire simple a été utilisé pour chaque province et chaque capitale territoriale. Sur les 14689 personnes qui ont répondu au sondage, $84 \%$ ont accepté de partager leurs données avec l'ASPC. L'échantillon utilisé pour cette analyse compte donc 12344 personnes. Les personnes vivant dans une réserve ou d'autres établissements autochtones, les membres à temps plein des Forces armées canadiennes et les personnes incarcérées sont exclues du champ de l'étude. Davantage de précisions sur le plan d'échantillonnage et le cadre d'échantillonnage de l'ECSM sont disponibles sur le site Internet de Statistique Canada ${ }^{13}$.

\section{Changements autodéclarés relatifs à la consommation d'alcool et de cannabis}

Les questions suivantes ont été posées aux participants : "Comment votre consommation d'alcool a-t-elle changé durant la pandémie de COVID-19 par rapport à avant? » et « Comment votre consommation de cannabis a-t-elle changé durant la pandémie de COVID-19 par rapport à avant? ». Les choix de réponses étaient les suivants : "Augmentation », "Diminution » et " Aucun changement ». Les répondants ayant oublié ou refusé de répondre à ces questions ont été exclus de l'analyse et leurs données ont été considérées comme manquantes ( $\mathrm{n}=43)$.

\section{Analyse des données}

Nous avons estimé la prévalence pondérée (avec un intervalle de confiance de $95 \%$ [IC]) des déclarations 1) de hausse, 2) de baisse et 3) d'absence de changement relativement à la consommation d'alcool et de cannabis pour les répondants de 18 ans et plus. Les estimations portant sur la hausse autodéclarée de consommation ont été ventilées en fonction des facteurs suivants : genre, groupe d'âge, changements relatifs au revenu depuis le début de la pandémie de COVID-19 (augmentation, diminution, aucun changement), quintile du revenu total du ménage déclaré, nombre de personnes composant le ménage, être parent ou tuteur légal d'un ou de plusieurs enfants de moins de 18 ans, niveau de scolarité (sans diplôme d'études secondaires, diplôme d'études secondaires, diplôme d'études postsecondaires), s'auto-identifier comme membre d'un groupe dit " racialisé » (oui ou non), statut vis-à-vis de l'immigration, province et capitale territoriale, milieu de résidence (urbain ou rural) et changements déclarés sur le plan de la santé mentale par rapport à avant la pandémie de COVID-19.

Les poids d'échantillonnage ont été fournis par Statistique Canada dans le but de générer des estimations représentatives à l'échelle nationale. La variance a été estimée à l'aide de la méthode bootstrap. Les analyses statistiques ont été réalisées à l'aide de la version 7.1 de SAS Enterprise Guide (SAS Institute Inc, Cary, Caroline du Nord, États-Unis). Des tests du chi carré ont aussi été effectués pour analyser les associations significatives entre les variables sociodémographiques, la santé mentale audéclarée et les comportements relatifs à la consommation d'alcool et de cannabis.

\section{Résultats}

D’après des données représentatives à l'échelle nationale, 15,7\% des répondants ont déclaré avoir augmenté leur consommation d'alcool, 9,9\% l'avoir diminuée et
74,3 \% n'avoir remarqué aucun changement. Pour le cannabis, 5,4 \% des répondants ont déclaré avoir augmenté leur consommation, 1,8 \%, l'avoir diminuée, $19,5 \%$ n'avoir remarqué aucun changement et 73,3 \% n'avoir jamais consommé de cannabis. Les estimations de la prévalence liée à l'augmentation de la consommation d'alcool ou de cannabis varient lorsque les données sont ventilées. Les différences statistiquement significatives sont mises en évidence dans la section "Résultats » et les estimations sont présentées dans les tableaux 1 et 2 .

\section{Consommation d'alcool}

La prévalence associée à une consommation autodéclarée accrue d'alcool est plus élevée chez les 35 à 44 ans (21,9\%) et les 45 à 54 ans $(21,0 \%)$, chez les répondants ayant un diplôme d'études postsecondaires $(18,4 \%)$ et chez ceux ayant déclaré avoir vécu des changements concernant le revenu de leur ménage depuis le début de la pandémie de COVID-19 (18,5 \% pour les personnes dont le revenu a augmenté et $19,1 \%$ pour les personnes dont le revenu a diminué). Cette prévalence augmente avec celle du quintile de revenu (de $7,5 \%$ à 27,2 \%). La capitale des Territoires du Nord-Ouest, Yellowknife, a la plus forte prévalence d'augmentation autodéclarée de consommation d'alcool (22,5 \%), l'îledu-Prince-Édouard ayant la plus faible (11,0 \%). Le pourcentage d'augmentation autodéclarée de consommation d'alcool est plus élevé chez les personnes vivant en milieu urbain (16,2\%), chez celles vivant dans un ménage de quatre personnes $(20,8 \%)$, chez les parents ou les tuteurs légaux d'enfants de moins de 18 ans $(22,6 \%)$, chez les natifs du Canada (" non-immigrants ») (18,2\%) et les chez personnes qui n'estiment pas faire partie d'un groupe dit "racialisé » $(17,8 \%)$. Enfin, le taux d'augmentation de la consommation d'alcool le plus élevé se retrouve chez les personnes ayant déclaré que leur santé mentale était bien moins bonne ou un peu moins bonne aujourd'hui qu'avant la pandémie de COVID-19 (27,0 \%).

\section{Consommation de cannabis}

La prévalence d'une consommation autodéclarée accrue de cannabis est plus élevée chez les hommes $(5,8 \%)$, les non-immigrants $(6,3 \%)$, les personnes ayant déclaré que leur revenu avait diminué depuis le début de la pandémie de COVID-19 (7,9 \%) et les personnes ayant 
déclaré que leur santé mentale était bien moins bonne maintenant ou un peu moins bonne maintenant (10,0 \%). L'augmentation autodéclarée de consommation de cannabis diminue avec l'âge (de 12,1 \% à $1,0 \%)$. La plus élevée est en NouvelleÉcosse $(7,8 \%)$ et la plus faible en Saskatchewan (3,0\%).

\section{Analyse}

Globalement, 15,7 \% des personnes vivant au Canada ont déclaré avoir augmenté leur consommation d'alcool et 5,4\% leur consommation de cannabis au cours de la deuxième vague de la pandémie de COVID-19. Ces données ont été ventilées en fonction des caractéristiques sociodémographiques et des changements déclarés concernant la santé mentale, ce qui a permis d'obtenir des données supplémentaires sur les répercussions générales de la pandémie. Les résultats obtenus pour la consommation de cannabis concordent avec l'augmentation signalée au début de la pandémie, soit du 29 mars au 3 avril 2020 (première vague), dans le cadre de la série d'enquêtes sur les perspectives canadiennes (SEPC) $(5,5 \%)^{10}$. Notre estimation de l'augmentation de la consommation d'alcool est légèrement supérieure à celle obtenue au terme de la série 1 de la SEPC $(14 \%)^{10}$.

Après ventilation des données, les résultats diffèrent en fonction des variables sociodémographiques, ce qui laisse penser qu'il existerait des disparités pour certains groupes. L'augmentation de la consommation d'alcool et de cannabis varie considérablement en fonction du groupe d'âge, de la province et des changements relatifs au revenu du ménage depuis le début de la pandémie de COVID-19. Fait notable, le nombre de personnes ayant déclaré avoir augmenté leur consommation d'alcool augmente avec l'âge, tandis que l'augmentation de la consommation de cannabis semble diminuer avec l'âge. De plus, les personnes ayant auto-évalué leur santé mentale comme bien moins bonne ou un peu moins bonne qu'avant la pandémie de la COVID-19 ont davantage tendance à avoir augmenté leur consommation d'alcool $(27 \%)$ et de cannabis (10\%) que celles estimant au moment de l'enquête que leur santé mentale était à peu près la même, un peu meilleure ou bien meilleure qu'avant la pandémie. Ce résultat coïncide avec ce qui est rapporté dans la littérature $^{4,11}$ et met en évidence la relation
TABLEAU 1

Caractéristiques des répondants de l'étude sur les changements relatifs à la consommation d'alcool et de cannabis depuis le début de la pandémie de COVID-19, Canada, septembre à décembre 2020

\begin{tabular}{lr} 
Variable & $\begin{array}{c}\text { Proportion, en } \% \\
\text { (IC à } 95 \%)\end{array}$ \\
\hline Genre & $49,1(48,9$ à 49,3$)$ \\
\hline Hommes & $50,7(50,6$ à 50,7$)$ \\
Femmes & \\
\hline Groupe d'âge (ans) & $9,5(8,6$ à 10,4$)$ \\
18 à 24 & $18,7(17,8$ à 19,6$)$ \\
25 à 34 & $16,8(16,8$ à 16,8$)$ \\
35 à 44 & $15,6(15,6$ à 15,6$)$ \\
45 à 54 & $17,2(17,2$ à 17,2$)$ \\
55 à 64 & $22,2(22,2$ à 22,2
\end{tabular}

Changements relatifs au revenu depuis le début de la pandémie de CovID-19

Augmentation

$6,0(5,3$ à 6,7$)$

Diminution

$37,5(36,3$ à 38,8$)$

Aucun changement

$56,5(55,2$ à 57,7$)$

Quintile du revenu total du ménage déclaré

Q1

$21,1(20,0$ à 22,2)

Q2

$20,2(19,1$ à 21,3$)$

Q3

$22,4(21,2$ à 23,6$)$

Q4

$18,3(17,2$ à 19,4$)$

Q5

$18,0(16,9$ à 19,1$)$

Province/capitale territoriale

Colombie-Britannique

$13,4(13,4$ à 13,4$)$

Alberta

$11,4(11,4$ à 11,4$)$

Saskatchewan

$2,8(2,8$ à 2,8$)$

Manitoba

3,3 (3,3 à 3,3)

Ontario

39,5 (39,5 à 39,5)

Québec

$22,9(22,9$ à 22,9)

Nouveau-Brunswick

$2,0(2,0$ à 2,0$)$

Nouvelle-Écosse

$2,6(2,6$ à 2,6)

Île-du-Prince-Édouard

$0,4(0,4$ à 0,4$)$

Terre-Neuve-et-Labrador

$1,4(1,4$ à 1,4$)$

Whitehorse (Yukon)

$0,1(0,1$ à 0,1$)$

Yellowknife (Territoires du Nord-Ouest)

$0,1(0,1$ à 0,1$)$

Iqaluit (Nunavut)

$0,02(0,02$ à 0,02$)$

\section{Milieu de résidence}

Urbain

$82,3(81,5$ à 83,1$)$

Rural

$17,7(16,9$ à 18,5$)$

Nombre de personnes dans le ménage

1

$14,5(14,1$ à 14,9$)$

2

$35,5(34,8$ à 36,2$)$

3

$17,5(16,5$ à 18,5$)$

4

$18,6(17,4$ à 19,7$)$

5 ou plus

$13,9(12,7$ à 15,1$)$

Suite à la page suivante 
TABLEAU 1 (suite)

Caractéristiques des répondants de l'étude sur les changements relatifs à la consommation d'alcool et de cannabis depuis le début de la pandémie de COVID-19, Canada, septembre à décembre 2020

\begin{tabular}{|c|c|}
\hline Variable & $\begin{array}{l}\text { Proportion, en \% } \\
\text { (IC à } 95 \% \text { ) }\end{array}$ \\
\hline \multicolumn{2}{|c|}{ Parent ou tuteur légal d'un ou de plusieurs enfants de moins de 18 ans } \\
\hline Oui & $27,6(26,6$ à 28,5$)$ \\
\hline Non & $72,4(71,5$ à 73,4) \\
\hline \multicolumn{2}{|l|}{ Niveau de scolarité } \\
\hline Sans diplôme d'études secondaires & $7,6(6,9$ à 8,3) \\
\hline Diplôme d'études secondaires & $23,6(22,4$ à 24,8$)$ \\
\hline Diplôme d'études postsecondaires & $68,8(67,5$ à 70,0$)$ \\
\hline \multicolumn{2}{|c|}{ Personnes qui s'auto-identifient comme membres d'un groupe « racialisé » } \\
\hline Oui & $24,3(23,1$ à 25,4$)$ \\
\hline Non & $75,7(74,6$ à 76,9$)$ \\
\hline \multicolumn{2}{|l|}{ Immigrant } \\
\hline Oui & $25,6(24,4$ à 26,8$)$ \\
\hline Non & $73,0(71,8$ à 74,1$)$ \\
\hline Résident non permanent & $1,4^{\mathrm{E}}(1,1$ à 1,8$)$ \\
\hline \multicolumn{2}{|c|}{ Santé mentale autodéclarée par rapport à la situation avant la pandémie de COVID-19 } \\
\hline Bien meilleure maintenant / un peu meilleure maintenant & $7,6(6,8$ à 8,3$)$ \\
\hline À peu près la même & $59,0(57,6$ à 60,3$)$ \\
\hline Bien moins bonne / un peu moins bonne & $33,5(32,2$ à 34,8$)$ \\
\hline \multicolumn{2}{|l|}{ Changements autodéclarés relatifs à la consommation d'alcool } \\
\hline Augmentation & $15,7(14,7$ à 16,7$)$ \\
\hline Diminution & $9,9$ (9,1 à 10,8$)$ \\
\hline Aucun changement & $74,3(73,2$ à 75,5$)$ \\
\hline \multicolumn{2}{|c|}{ Changements autodéclarés relatifs à la consommation de cannabis } \\
\hline Augomentation & $5,4(4,8$ à 6,1$)$ \\
\hline Diminution & $1,8(1,4$ à 2,1$)$ \\
\hline Aucun changement & $19,5(18,4$ à 20,5$)$ \\
\hline N’a jamais consommé de cannabis & 73,3 (72,1 à 74,5) \\
\hline
\end{tabular}

Source des données : Enquête sur la COVID-19 et la santé mentale de 2020.

Abréviations : IC, intervalle de confiance; Q, quintile.

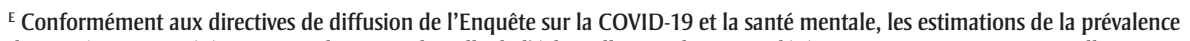
doivent être interprétées avec prudence, car la taille de l'échantillon total non pondéré se situe entre 75 et 150 . Veuillez tenir compte des intervalles de confiance lorsque vous interprétez ces estimations.

complexe entre santé mentale et consommation d'alcool et de cannabis.

Nous avons également constaté des différences en fonction des substances. Chez les personnes ayant déclaré avoir augmenté leur consommation d'alcool, des différences substantielles ont été relevées en fonction du quintile du revenu total du ménage, du milieu de résidence (urbain ou rural), de la taille et de la composition du ménage ainsi que de l'appartenance autodéclarée à un groupe " racialisé ». Il est intéressant de noter qu'aucune différence entre les genres n’a été observée relativement à la consommation d'alcool.
En proportion, davantage d'hommes que de femmes ont déclaré avoir augmenté leur consommation de cannabis parmi les répondants ayant déclaré une augmentation de cette consommation.

Il est important de noter que ces estimations sont représentatives de l'ensemble de la population observée, incluant celle n'ayant jamais consommé d'alcool ou de cannabis. Ces résultats seraient probablement considérablement différents si notre échantillon se limitait aux répondants ayant déjà consommé ces substances. Comme le Canada est actuellement à l'étape de la relance, les études à venir devraient continuer de surveiller la consommation d'alcool et de cannabis à l'échelle de l'ensemble de la population, y compris chez les non-consommateurs. Toutefois, on devrait également étudier plus spécifiquement les personnes consommant ces substances afin d'orienter les stratégies de prévention et de réduction des méfaits en matière de santé publique. Il serait également justifié de mener des recherches pour étudier l'effet de certaines mesures susceptibles d'avoir contribué à l'augmentation de la consommation d'alcool et de cannabis (par exemple la livraison à domicile d'alcool).

\section{Conclusion}

Au cours de la deuxième vague de la pandémie de la COVID-19 au Canada, environ $15,7 \%$ des Canadiens ont déclaré avoir augmenté leur consommation d'alcool et $5,4 \%$ leur consommation de cannabis. Ces estimations représentatives à l'échelle nationale varient en fonction des caractéristiques sociodémographiques, ce qui met en évidence la nécessité de comprendre les déterminants sociaux de la santé. Les études à venir devraient porter sur le lien entre les déterminants et la consommation de substances pendant la pandémie de COVID-19 afin de déterminer quelles populations à risque pourraient tirer profit d'une sensibilisation accrue, d'efforts d'atténuation et de ressources relatives aux méfaits liés à l'alcool et au cannabis.

\section{Remerciements}

Les auteures désirent remercier Lil Tonmyr (de l'Agence de la santé publique du Canada) et Statistique Canada pour leur contribution à la conception de l'Enquête sur la COVID-19 et la santé mentale. Elles remercient également Jeyasakthi Venugopal (Agence de la santé publique du Canada) d'avoir vérifié l'analyse et les résultats obtenus. Merci également au personnel de Statistique Canada et du Programme d'accès et de coordination des données (PACD) de l'Agence de la santé publique du Canada pour leur aide en ce qui concerne la diffusion des données. Nous tenons à remercier toutes les personnes de Statistique Canada qui ont participé à la collecte des données. Enfin, nous remercions toutes les personnes qui ont participé à cette enquête.

\section{Conflits d'intérêts}

Les auteures déclarent n'avoir aucun conflit d'intérêts. 
TABLEAU 2

Proportions d'augimentation autodéclarée de consommation d'alcool et de consommation de cannabis depuis le début de la pandémie de COVID-19, en fonction des caractéristiques sociodémographiques, adultes (18 ans et plus), Canada, septembre à décembre 2020

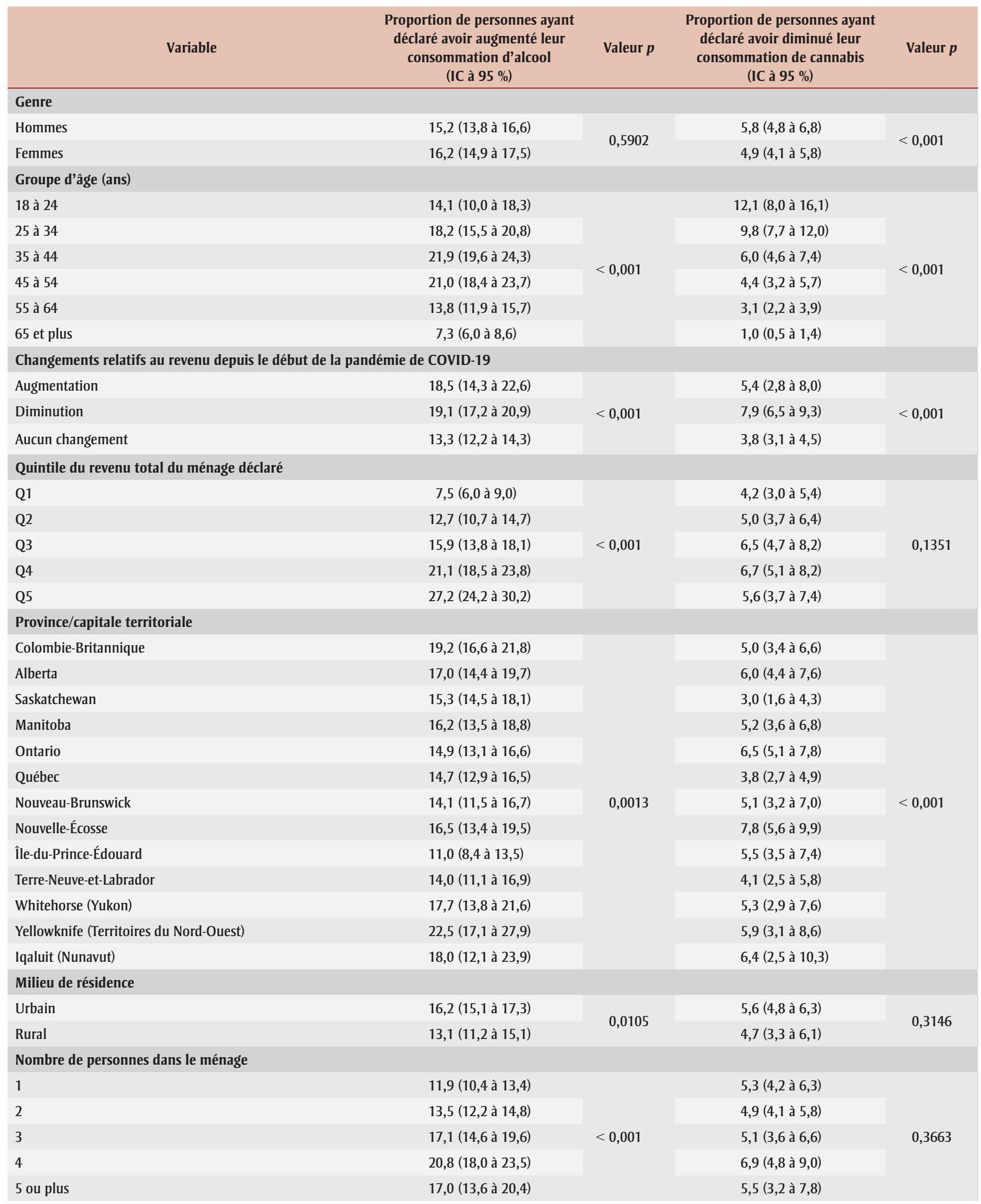


TABLEAU 2 (suite)

Proportions d'augimentation autodéclarée de consommation d'alcool et de consommation de cannabis depuis le début de la pandémie de COVID-19, en fonction des caractéristiques sociodémographiques, adultes (18 ans et plus), Canada, septembre à décembre 2020

\begin{tabular}{|c|c|c|c|c|}
\hline Variable & $\begin{array}{l}\text { Proportion de personnes ayant } \\
\text { déclaré avoir augomenté leur } \\
\text { consommation d'alcool } \\
\text { (IC à } 95 \%)\end{array}$ & Valeur $p$ & $\begin{array}{l}\text { Proportion de personnes ayant } \\
\text { déclaré avoir diminué leur } \\
\text { consommation de cannabis } \\
\text { (IC à } 95 \% \text { ) }\end{array}$ & Valeur $p$ \\
\hline \multicolumn{5}{|c|}{ Parent ou tuteur légal d'un ou de plusieurs enfants de moins de 18 ans } \\
\hline Oui & $22,6(20,6$ à 24,5$)$ & \multirow{2}{*}{$<0,001$} & 4,8 (3,8 à 5,7) & \multirow{2}{*}{0,1749} \\
\hline Non & $13,1(12,0$ à 14,2$)$ & & $5,7(4,8$ à 6,5$)$ & \\
\hline \multicolumn{5}{|l|}{ Niveau de scolarité } \\
\hline Sans diplôme d'études secondaires & $4,9(3,2$ à 6,6$)$ & \multirow{3}{*}{$<0,001$} & $3,6(0,9$ à 6,3$)$ & \multirow{3}{*}{0,3849} \\
\hline Diplôme d'études secondaires & $11,5(9,5$ à 13,5$)$ & & 5,3 (3,9 à 6,7) & \\
\hline Diplôme d'études postsecondaires & $18,4(17,2$ à 19,6$)$ & & 5,7 (4,9 à 6,5) & \\
\hline \multicolumn{5}{|c|}{ Personnes qui s'auto-identifient comme membres d'un groupe « racialisé » } \\
\hline Oui & $9,4(7,4$ à 11,4$)$ & \multirow{2}{*}{$<0,001$} & $5,1(3,5$ à 6,8$)$ & \multirow{2}{*}{0,6659} \\
\hline Non & 17,8 (16,7 à 18,9) & & $5,5(4,8$ à 6,2$)$ & \\
\hline \multicolumn{5}{|l|}{ Immigrant } \\
\hline Oui & 8,8 (7,3 à 10,4) & \multirow{3}{*}{$<0,001$} & $3,1(1,8$ à 4,4$)$ & \multirow{3}{*}{0,0024} \\
\hline Non & $18,2(17,1$ à 19,4$)$ & & $6,3(5,5$ à 7,1$)$ & \\
\hline Résident non permanent & $14,5^{\mathrm{E}}(2,4$ à 26,6$)$ & & $6,6^{\mathrm{E}}(0,2$ à 12,9$)$ & \\
\hline \multicolumn{5}{|c|}{ Santé mentale autodéclarée par rapport à la situation avant la pandémie de COVID-19 } \\
\hline Bien meilleure maintenant / un peu meilleure maintenant & $13,6(10,3$ à 16,9$)$ & \multirow{3}{*}{$<0,001$} & 8,8 (5,0 à 12,6) & \multirow{3}{*}{$<0,001$} \\
\hline À peu près la même & $9,5(8,6$ à 10,5$)$ & & $2,4(1,8$ à 3,0$)$ & \\
\hline Bien moins bonne / un peu moins bonne & $27,0(24,9$ à 29,1$)$ & & $10,0(8,5$ à 11,5$)$ & \\
\hline
\end{tabular}

Source des données : Enquête sur la COVID-19 et la santé mentale de 2020.

Abréviations : IC : intervalle de confiance; Q : quintile.

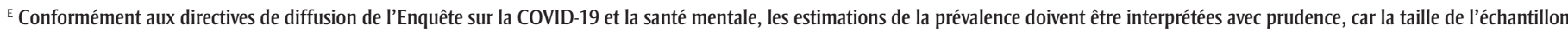
total non pondéré se situe entre 75 et 150 . Veuillez tenir compte des intervalles de confiance lorsque vous interprétez ces estimations.

\section{Contributions des auteures et avis}

MV, KHM, NH et MB ont rédigé l'article. MV s'est chargée des analyses statistiques. Toutes les coauteures ont interprété les données et révisé l'article.

Le contenu de l'article et les points de vue qui y sont exprimés n'engagent que les auteures; ils ne correspondent pas nécessairement à ceux du gouvernement du Canada.

\section{Références}

1. Commission de la santé mentale du Canada (CSMC), Nanos Research. Canadians report an increase in feeling stressed regularly or all the time now compared to one month before COVID-19. Ottawa (Ont.) : CSMC; 2020. En ligne à : https://www .mentalhealthcommission.ca/sites /default/files/2020-05/nanos_covid_ may_2020.pdf
2. Gadermann AC, Thomson KC, Richardson CG, et al. Examining the impacts of the COVID-19 pandemic on family mental health in Canada: findings from a national cross-sectional study. BMJ Open. 2021;11(1):e042871. https://doi.org/10.1136/bmjopen-2020 $-042871$

3. Hawke LD, Barbic SP, Voineskos A, et al. Impacts of COVID-19 on youth mental health, substance use, and well-being: a rapid survey of clinical and community samples. Can J Psychiatry. 2020;65(10):701-709. https://doi.org/10.1177\%2F07067437 20940562

4. Dozois DJ, Recherche en santé mentale Canada. Anxiety and depression in Canada during the COVID-19 pandemic: a national survey. Can Psychol. 2021;62(1):136-42. https://doi.org/10 $.1037 /$ cap0000251
5. Schmitz N, Holley P, Meng X, Fish L, Jedwab J. COVID-19 and depressive symptoms: a community-based study in Quebec, Canada. Can J Psychiatry. 2020;65(10):733-735. https://doi.org /10.1177/0706743720943812

6. Burns L, Teesson M. Alcohol use disorders comorbid with anxiety, depression and drug use disorders. Findings from the Australian National Survey of Mental Health and Well Being. Drug Alcohol Depend. 2002; 68(3):299-307. https://doi.org/10.1016 /s0376-8716(02)00220-x

7. Bolton J, Cox B, Clara I, Sareen J. Use of alcohol and drugs to self-medicate anxiety disorders in a nationally representative sample. J Nerv Ment Dis. 2006;194(11):818-825. https:// doi.org/10.1097/01.nmd.0000244481 .63148 .98 
8. Boschloo L, Vogelzangs N, Smit JH, et al. Comorbidity and risk indicators for alcohol use disorders among persons with anxiety and/or depressive disorders: findings from the Netherlands Study of Depression and Anxiety (NESDA). J Affect Disord. 2011;131(13):233-242. https://doi.org/10.1016/j .jad.2010.12.014

9. Schlossarek S, Kempkensteffen J, Reimer J, Verthein U. Psychosocial determinants of cannabis dependence: a systematic review of the literature. Eur Addict Res. 2016;22(3): 131-144. https://doi.org/10.1159 /000441777

10. Zajacova A, Jehn A, Stackhouse M, Denice P, Ramos H. Changes in health behaviours during early COVID-19 and socio-demographic disparities: a cross-sectional analysis. Can J Public Health. 2020;111(6):953962. https://doi.org/10.17269/s41997 $-020-00434-y$

11. Association canadienne pour la santé mentale (ACSM), University of British Columbia. Les conséquences de la COVID-19 sur la santé mentale : Vague 2. Toronto (Ont.) : ACSM; 2020. En ligne à : https://cmha.ca /wp-content/uploads/2021/07/ACSM -UBC-Vague-2-Resume-des-conclusions .pdf

12. Evans AC, Bufka LF. The critical need for a population health approach: addressing the nation's behavioral health during the COVID-19 pandemic and beyond. Prev Chronic Dis. 2020:17:200261. https://doi.org/10 $.5888 / \operatorname{pcd} 17.200261$

13. Statistique Canada. Enquête sur la COVID-19 et la santé mentale (ECSM) [Internet]. Ottawa (Ont.) : Statistique Canada; 2020. En ligne à : https:// www23.statcan.gc.ca/imdb/p2SV_f.pl ?Function $=$ getSurvey $\& I d=1283036$ 